\title{
LA GEOGRAFÍA DE EGIPTO EN DIODORO DE SICILIA
}

This paper attempts to show that the geographical chapters concerning Egypt in the first book of Diodorus' Bibliotheca are not to be explained, as it is usually done, from the viewpoint of an ethnographical utopia, but from that of the encomium of a country, recognized as such in ancient handbooks of Rhetoric. It also attempts to point out that the ultimate responsibility of these chapters must be ascribed to Diodorus himself, who has described the geography of Egypt in Book I in an encomiastic way quite coherent with the role that it plays in the following books of his Bibliotheca.

1. Es doctrina generalmente aceptada que Diodoro de Sicilia ha seguido fundamentalmente a Hecateo de Abdera en la elaboración de la parte egipcia de su Biblioteca Histórica, si bien con la importante restricción de que para los capítulos 32-41 hay que postular una fuente posterior o quizá más de una, dado que éstos contienen ciertos datos cuya adscripción a Hecateo es cronológicamente imposible. Esta communis opinio tiene como consecuencia nada satisfactoria tener que atribuir a dos fuentes distintas la exposición diodorea de la geografia egipcia, la cual, además de la sección arriba mencionada, abarca también los capítulos 30-31, cuya adscripción a Hecateo puede ser mantenida'.

1 Este trabajo forma parte del Proyecto de Investigación PB 88-0483 de la DGICYT que está desarrollando actualmente el grupo de trabajo "Historiografía Antigua" (n." 1062 PAI). Cf. A. Burton, Diodorus Siculus, Book I. A Commentary, Leiden 1972, p. 18 ss., quien, a su vez, contempla la posibilidad de una partición entre los capítulos 30-36, por una parte, y por otra, 37-41, concluyendo que para esta última sección se puede considerar la fuente última de Diodoro Agatárquides y la fuente inmediata Artemidoro, quien quizá sea también la fuente principal para los capitulos 30-36. En cualquier caso, el énfasis que habia puesto H. Leopoldi (De Agatharchide Cnidio, Diss. inaug., Rostock 1892, p. 21) en que Diodoro (I 39,7) no puede haber calificado de

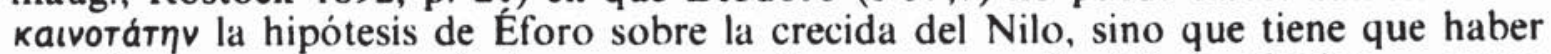
tomado esta expresión (y la argumentación correspondiente) de alguien - como Agatárquides - para quien Éforo estuviese próximo en el tiempo, se basa en una interpretación equivocada de каıvoт́́rqu como "la más reciente». A la argumentación de Burton (p. 22) de que la traducción correcta es "la más novedosa" cabe añadir 
El supuesto fundamental de esta argumentación es el postulado de que Diodoro no puede haber realizado por su cuenta la actualización que, respecto de Hecateo, presuponen partes de los arriba mencionados capítulos 32-41.

El problema de las fuentes, a su vez, se involucra con el de la interpretación de esta parte egipcia del libro I. Jacoby ${ }^{2}$ argumentó que Hecateo de Abdera había operado una alteración importante en la fórmula habitual del logos etnográfico conforme a unos intereses que ya no eran los de la historiè jonia, sino los de un filósofo que se habría servido de un género histórico para sus fines especulativos y protrépticos. Hecateo estaba interesado en presentar un estado ideal en la tradición del pensamiento político del siglo iv que añadiria a su excelencia el carácter «real» de un país existente. El desplazamiento de los contenidos geográficos, junto con los propiamente históricos, sería para Jacoby el resultado inexcusable de esa transformación que convierte a Hecateo en el creador de un nuevo género: la "utopía etnográfica».

Las ideas de Jacoby han sido desarrolladas por Oswyn Murray. Este autor insiste en que, entre los intereses de Hecateo al escribir su obra sobre Egipto, la cuestión geográfica tenía un lugar bastante secundario $^{3}$. A Hecateo remontarian los capítulos 30 y 31 del libro I de Diodoro, en los que se ofrece primero una somera descripción de las fronteras de Egipto, que garantizan al país una protección privilegiada, y a continuación los datos sobre la abundancia de población, que conectarian adecuadamente los theologoúmena con la sección histórica.

La transformación más significativa de la parte mencionada del libro I, supuestamente derivada de Hecateo, con respecto a la tradición que para nosotros inaugura el $\log o s$ de Heródoto sobre Egipto estribaría, pues, en la pérdida de relevancia de la parte geográfica en el plan de la obra; en efecto, la importancia de la especulación sobre las peculiaridades geográficas del país del Nilo en el libro II de las Historias de Heródoto, tanto si se considera desde la perspectiva de la evolución in-

el testimonio de Elio Aristides, quien (jen el siglo II d.C.!) designa la hipótesis de

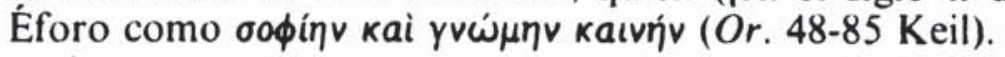

2 FGrHist III A Comm., Leiden 1969, pp. 34 s., 79. Sobre la estructura del logos etnográfico, véase, del mismo autor, «Über die Entwicklung der griechischen $\mathrm{Hi}$ storiographie und den Plan einer neuen Sammlung der griechischen Historikerfragmente», en Abhandlungen zur griechischen Geschichtsschreibung, Leiden 1956, p. 26 ss. Para la orientación filosófica de Hecateo y su aprovechamiento de la tradición etnográfica, cf. R. Drews, The Greek accounts of Eastern history, Washington 1973, p. 122 ss.

3 "Hecataeus of Abdera and Pharaonic Kingship", JEA 56, 1970, p. 141 ss., en especial $147 \mathrm{~s}$. 
telectual de su autor como si se hace atendiendo a la economía de la obra en su conjunto, es un punto en el que coincide la rara vez unánime investigación sobre el historiador de Halicarnaso.

Por tanto, la ausencia en la obra de Hecateo de un tratamiento satisfactorio de temas tan atractivos para el público griego como el Nilo y sus crecidas habría obligado a Diodoro a buscar su información en un autor como Agatárquides, más pródigo en estos temas.

Murray subraya que lo definitorio del Egipto de Hecateo, la fortificación natural que le ofrecen los accidentes naturales que lo circundan, es una presentación asociada al carácter ideal del país del Nilo. Gracias a esta referencia a la inaccesibilidad de Egipto, esta tierra adquiere la lejanía característica de la utopía: «Sus datos, de hecho, no hacen más que subrayar la fortaleza de las fronteras de Egipto, su aislamiento y su adecuación al estado filosóficamente ideal, carente de contacto con la corrupción de sus vecinos y completamente autosuficiente - la autárkeia ética de la escuela de Abdera reforzaba la importancia que a la autárkeia política otorgaba la filosofía política del siglo IV" ${ }^{4}$. Murray, con todo, añadía consideraciones más pragmáticas: la insistencia en la independencia geográfica de Egipto puede reflejar la perspectiva de Ptolomeo, interesado por razones obvias en subrayar la autonomía del país.

Zambrini, por su parte ${ }^{5}$, dio un paso más y se preguntó si la presentación que hace Hecateo de un Egipto totalmente aislado y defendido por la naturaleza no había influido en la presentación de la India como zona inaccesible a cargo de Megástenes. La extensión que hace Zambrini de la sugerencia de Murray es claramente abusiva: aunque se subraya la magnitud de la India, de la descripción megasténica de sus límites, de hecho, está totalmente ausente la mención de las defensas naturales ${ }^{6}$.

La propia interpretación dada por Murray de los capítulos 30-31 del

4 Art. cit., p. 148.

A. Zambrini, "Gli Indiká di Megastene, I", ASNP 12, 1982, pp. 71-149, esp. pp. 97-101 y 140-8. Zambrini precisa el alcance y los limites de esta influencia, señalados ya por Jacoby y Murray.

"Diodoro II 35. Zambrini ("Gli Indiká di Megastene, II", ASNP 15, 1985, p. 830) se cuestiona «se i confini geografici descritti de Megastene non contribuissero a dare un'immagine dell'India como zona inaccessibile, protetta com'è dall'Indo, dal Caucaso e dall'Oceano, cioè se in definitiva no vi fosse, anche a livello geografico, una rippresa del motivo di Ecateo di Abdera di un Egitto totalmente isolato e naturalmente munito". Zambrini da por seguro que la descripción de la fortificación natural de Egipto procede de Hecateo de Abdera. Pasa por alto, además, que lo que diferencia la descripción de los dos paises en cuestión es precisamente el hecho de que en el caso de la India falte por completo la indicación de que estos límites fortifican de modo natural la tierra. 
libro I de Diodoro plantea más dificultades que las que resuelve. Ya de entrada, no deja de sorprender la adscripción a Hecateo de intenciones tan poco coherentes como la de atribuir a Egipto una inaccesibilidad "sanitaria», que lo preservaría de la corrupción de sus vecinos, y la muy concreta instrumentalización de estas circunstancias por parte del fundador de la dinastía ptolemaica ${ }^{7}$.

En cuanto a la noción de las formidables defensas naturales de Egipto, que ocupa un lugar sustancial en los capitulos en cuestión, hay que observar que el énfasis no está puesto tanto en la inaccesibilidad como en la consideración del país como aquel al que la naturaleza ha proporcionado una notable autodefensa. Si ya desde ahora tomamos en consideración el resto del libro I, encontraremos que existe a este respecto una notable coherencia, pues las portentosas murallas naturales no impiden el contacto con pueblos diversos, y no hay constatación de que el acceso de los extranjeros al país a partir del reinado de Psamético comportara degeneración o decadencia ${ }^{8}$.

Con carácter más general, si admitimos que Heródoto es heredero de una considerable tradición científica que encuentra en Egipto un enclave privilegiado para sus indagaciones, y, a su vez, que su logos egipcio, en cuanto plasmación articulada y completa de los contenidos relativos a un país susceptibles de provocar interés, ha venido a ser modelo de primer orden para la literatura etnográfica griega - modelo que experimentó en época helenística un notable desarrollo ${ }^{9}-$, se hace difícil

${ }^{7}$ Esta segunda noción no se deduce ni del conjunto de I 30-31 ni de una parte

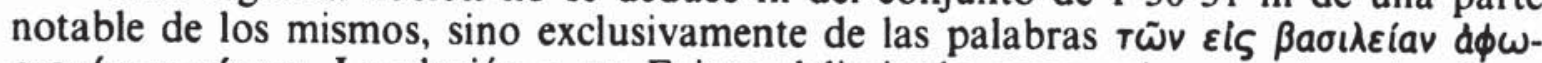

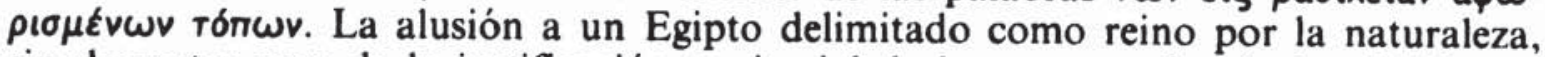
sin descartar ecos de la justificación territorial de las monarquías de los Diádocos, puede ser vista también como elemento integrante de la loa de un país; cf. D.C.,

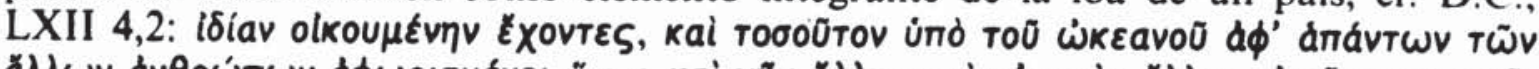

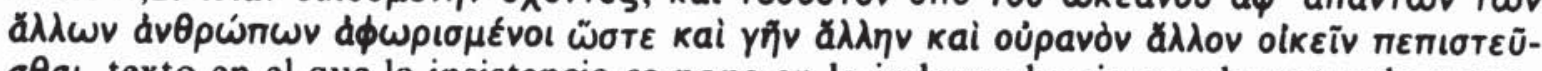
$\sigma \theta a i$, texto en el que la insistencia se pone en la independencia que la naturaleza proporciona a Bretaña, garantizándole así su identidad.

${ }^{8}$ Por lo mismo, no podemos estar de acuerdo con Murray cuando argumenta (art. cit., p. 147) que hay una frase en I 31,9 que sugiere transición a la parte de exposición histórica, pero que inmediatamente Diodoro la pospone para insertar una larga digresión sobre el Nilo. No creemos que a nivel textual I 31,9 marque la fractura que permitiría detectar el cambio de fuente, sino que, al contrario, en I 29,6

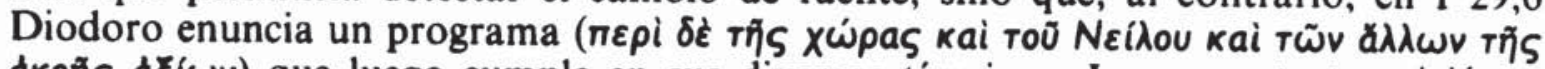
dkoñ $\varsigma$ $\phi \zeta ̧(\omega v)$ que luego cumple en sus diversos términos. La presunta transición a la historia en I 31,9 que se reduce a un par de líneas, es preferible leerla como un

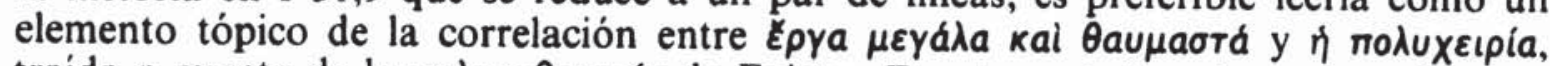

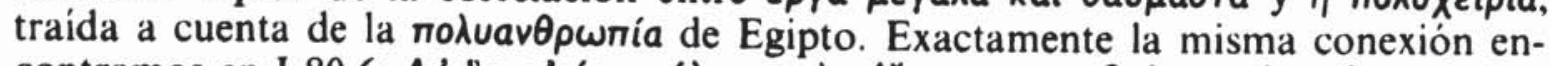

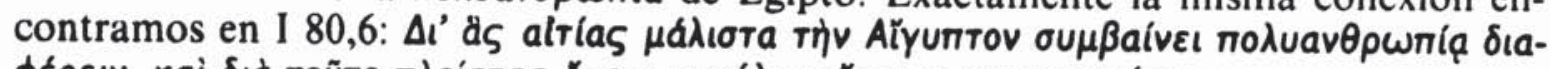

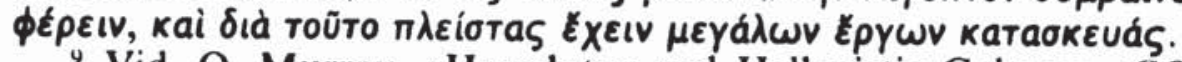
213.

"Vid. O. Murray, "Herodotus and Hellenistic Culture», $C Q 60,1972$, pp. 200- 
admitir que Hecateo hubiese introducido, precisamente sobre Egipto, un desajuste tan radical que lo desnaturalizara, minusvalorando el componente geográfico e histórico hasta casi su eliminación.

En realidad, hay que afirmar que cuando Jacoby consagró con su autoridad la concepción de la obra de Hecateo sobre Egipto como "utopía etnográfica», introdujo un concepto que ha sido fuente de numerosos equívocos ${ }^{10}$.

2. Este trabajo pretende argumentar que los capitulos geográficos del libro I de la Biblioteca de Diodoro relativos a Egipto pueden ser explicados de un modo mucho más satisfactorio que a partir de la noción de "utopía etnográfica» partiendo de los esquemas retóricos de exaltación de un pais en general y de Egipto en particular. Aspira también a mostrar que la responsabilidad última de la redacción de dichos capítulos puede ser atribuida al propio Diodoro, quien ha realizado uno de dichos esquemas retóricos en el libro I de modo notablemente coherente con el papel que la geografia egipcia desempeñará en los libros posteriores de la Biblioteca.

La existencia de esquemas tópicos para la descripción de un país resulta particularmente clara a partir de la obra de Menandro Sobre los fines epidicticos, en la que se dedica una sección a cómo elogiar un país". Como nos recuerdan Russell y Wilson ${ }^{12}$, los rétores tenían a su disposición, además de la lírica arcaica ${ }^{13}$, buena cantidad de material de

${ }^{10}$ Sin entrar ahora en una discusión detenida del concepto mismo de "utopía etnográfica" que Jacoby también utilizó, por ejemplo, para la obra de Onesícrito sobre la India -, su aplicación a la obra de Hecateo de Abdera sobre Egipto plantea dificultades. Asi R. Drews (op. cit., p. 122 ss.), al interpretar las Aegyptiaca en la línea de Jacoby, ha de constatar su excepcionalidad. Otra excepcionalidad que se deriva de tal interpretación la constata G. J. D. Aalders, Political Thought in Hellenistic Times, Amsterdam 1975, pp. 64-65.

"Nos referimos al Tratado primero, atribuido a Genetlio de Petra (Spengel III 331-67); cf. L. Pernot, "Topique et topographie: l'espace dans la rhétorique épidictique grecque à l'époque impériale", en Ch. Jacob \& F. Lestringant (edd.), Arts et légendes d'espaces, París 1981, pp. 101-109, en particular 101; H. G. Downey, s.u. "Ekphrasis", RAC IV, 1959, col. 927, con bibliografia.

12 Menander Rhetor, Oxford 1981, p. 245.

${ }_{13}$ Los tópicos del encomio de ciudades y países han sido estudiados para la poesía antigua por E. Kienzle, Der Lobpreis von Städten und Ländern in den ältern griechischen Dichtung. Basilea 1936 (no hemos podido consultar la tesis de H. Gruber Der Lohpreis von Städten und Ländern in der griechischen Dichtung der alexandrinischen Zeit, Diss. Graz 1939). Aunque Kienzle no distingue la fortificación como tópico específico, los datos que recopila permiten reconocerlo al menos en tres aspectos del encomio: el de la situación natural, donde un buen número de epitetos indican la localización inaccesible; el de las edificaciones, con referencia a las murallas y los bastiones; finalmente, el tópico de los hombres como fortaleza, de tan gran impor- 
prosa clásica: el Panatenaico de Isócrates, los Ingresos de Jenofonte, el Critias platónico y las descripciones geográficas de los historiadores. Los elementos tópicos que con mayor frecuencia encontramos reiterados en dichas exposiciones laudatorias son la feracidad de la tierra y la excelencia de las defensas naturales. Nada de raro tiene el hecho de que sean precisamente éstos los dos puntos centrales, dado que responden perfectamente a los requisitos que para la prosperidad de un país exigian las condiciones socioeconómicas del mundo antiguo, en el cual la garantía de la independencia de un territorio dependía básicamente de su capacidad para asegurar su alimentación y su defensa.

El primer punto se encuentra con frecuencia desdoblado en un desarrollo principal sobre la productividad de la tierra y uno subsidiario sobre la abundante población que este hecho tiene como consecuencia. En cuanto al segundo, lo encontramos de un modo particularmente conciso y nítido en Quintiliano (III 7,26): Laudantur autem urbes similiter atque homines. Nam pro parente est conditor, et multum auctoritatis adfert uetustas, ut iis, qui terra dicuntur orti, et uirtutes ac uitia circa res gestas eadem quae in singulis: illa propria, quae loci positione ac munitione sunt. Ciues illis ut hominibus liberi sunt decori. Con respecto al tópico de la "posición estratégica», que es el que atrae especialmente a Diodoro, es interesante un pasaje de Menandro Rétor (Spengel III 349): 'ETı $\delta \dot{\varepsilon} \delta \psi \delta ́$ -

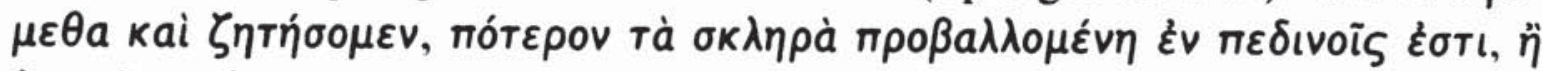

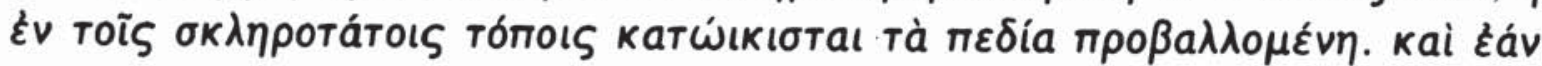

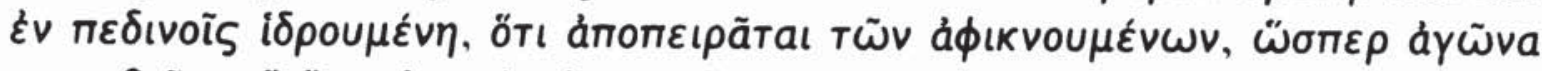

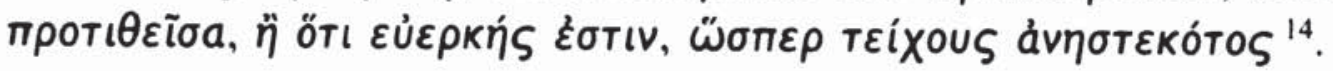

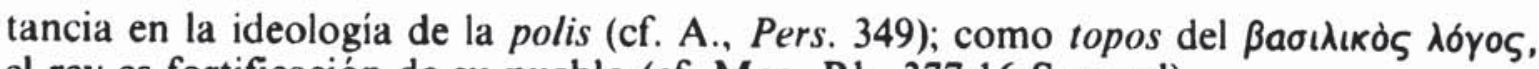
el rey es fortificación de su pueblo (cf. Men. Rh. 377,16 Spengel).

${ }_{14}$ Puede parecer impropio aplicar a un país el esquema de las laudes urbium. La aparente inadecuación, sin embargo, puede resolverse si leemos atentamente algunas referencias antiguas a Egipto. En efecto, en un texto tan difundido en su tiempo como la novela de Alejandro no ha dejado de causar perplejidad el hecho de que el autor, en el episodio de Alejandro en Menfis, hable de Egipto como si de una ciudad se tratara (I 37; análogamente en el caso de Persia: cf. II 13,1; 14,4). En el mismo Diodoro, como veremos más adelante, ambos términos, Egipto y Menfis, son presentados en una cierta correspondencia entre la simetría y la analogía. Pero tenemos que tener en cuenta sobre todo que, como ha indicado Martin (Antike Rhetorik. Technik und Methode, Munich 1974, p. 185), cuando Isócrates presenta en su Busiris la imagen idealizada de Egipto, lo hace en términos semejantes a los que ya eran habituales para el Ática, una tierra en la que es dificil distinguir a la hora del encomio territorio de polis. Por otro lado, el encomio de ciudades se concibe en los tratadistas como un género mixto que aprovecha y rearticula los tópicos laudatorios de otros géneros epidícticos fundamentales: los encomios de hombres y de lugares. Fórmula recurrente en la organización de los diferentes topoi es la distinción entre el placer

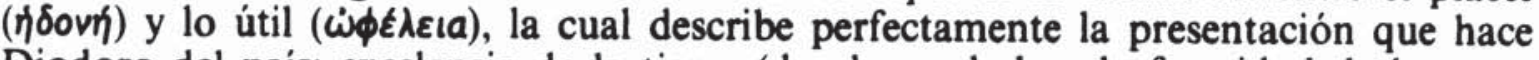
Diodoro del pais: excelencia de la tierra (donde se alude a la feracidad de los pro- 
El caso de la Atlántida en el Critias platónico es un caso complejo y especialmente desorientador, dado que, aunque presenta con bastante nitidez los dos componentes básicos del esquema, la próspera naturaleza del territorio y el carácter imponente de los accidentes naturales que lo circundan ${ }^{15}$, el valor de ambos tópicos no es en absoluto asimilable al que encontramos en el caso del Egipto diodoreo: el territorio era de extraordinaria feracidad y belleza $(114 \mathrm{e}, 118 \mathrm{~b}$ ), así como muy alto y escarpado (118 a). No obstante, no existe referencia alguna a que estas características geográficas tengan función defensiva. Por su parte, la construcción del primer recinto del palacio de los Atlantes por obra de Posidón muestra la confluencia de los motivos de abundancia y fortificación, pero la función de esa fortificación es que el territorio elegido sea inaccesible (ábaton) a los hombres por su carácter sagrado ${ }^{16}$.

En los relatos sobre los Aurea Saecula no se nos habla de las defensas (naturales o de otra indole), dado que, al no existir nada que provoque la codicia, no hay diferencias ni, en consecuencia, guerras ${ }^{17}$. En efecto, en el modelo de relato utópico que se toma cuando se habla del "aislamiento» y la "inaccesibilidad» de Egipto ${ }^{18}$ no hay habitualmente insistencia en la condición de lugar de fácil defensa, ya que la mayoría de las narraciones se refieren a localidades remotísimas, cuya existencia incluso se desconoce a veces, por lo que se recurre con frecuencia a la ficción de que son descubiertas accidentalmente en el curso de un viaje, o bien son localizadas, simplemente, en las partes más alejadas del mundo conocido. La situación en los confines de lo conocido es la traslación al espacio de la distancia que separa el mito de la historia ${ }^{19}$. Así,

ductos y, tal vez, a la belleza del paisaje, referidos ambos, en todo caso, al disfrute) y la protección natural que brinda; cf. Pernot, art. cit., pp. 102-103.

is Encontramos también en el caso de la Atlántida platónica un componente de la laus que reaparece con bastente frecuencia: gracias a su poder entran en el país bienes procedentes de lugares muy diversos (114 d). Es tema fundamental en el elogio de Roma por Elio Aristides, y aparecía ya entre las ventajas que su imperio reportaba a Atenas en el discurso de Pericles en el libro II de Tucidides y en el Pseudo-Je-

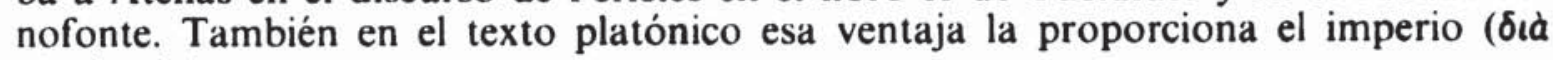
Tìv ápxív).

$16113 \mathrm{~d} ; 116 \mathrm{c}$.

17 Cf. L. Edelstein, The Idea of Progress in Classical Antiquity, Baltimore 1967. p. 134, quien cita a Dicearco (Porph., De Abstinentia IV 1, $2=$ F 49 Wehrli), según el cual en la edad de Crono los hombres no padecían guerras y disfrutaban, entre otros bienes, de salud, paz y amistad.

18 Para la complejidad del concepto de "utopía» y los diversos modelos implicados, cf. L. Bertelli, "Itinerari dell'utopia: della città ideale alle isole felice», en R. Ugliore (ed.), La città ideale nella tradizione classica e biblico-cristiana, Turín 1986, pp. 34-54 (con bibliografia), esp. 51 ss.

19 En efecto, tanto la inaccesibilidad del país mítico como su fertilidad sin parangón deben conectarse con las figuraciones del más allá, las Islas de los Bienaventura- 
por ejemplo, Ferguson ${ }^{20}$ hace notar que Escitobraquión sigue a Evémero en localizar su utopía en los límites del mundo conocido, pero, mientras que Evémero opta por el Este, él elige el Oeste. Es probable, admite Ferguson, que Escitobraquión tuviera la inquietud de la época por la circunnavegación de África; más probable es que no haga más que escoger una localización diametralmente opuesta a la usada por Evémero. $Y$ es que, en efecto, lo que en estos casos importa asegurar no es la «inatacabilidad» de ese país visto como bastión, sino su inaccesibilidad total, puesto que, debido a la ausencia de preparación militar de sus habitantes (entre cuyas características más reiteradas se cuenta la del pacifismo a ultranza), sólo una completa falta de acceso puede salvaguardar su existencia misma. Esta condición, al mismo tiempo, los preserva de la corrupción procedente del exterior.

Sobre los dos puntos, sin embargo, coincide la reflexión política cuando se ocupa de los requisitos que se han de atender para la fundación de una ciudad. Según Aristóteles ${ }^{21}$, son cuatro estos requisitos: la salubridad, la adecuación a las necesidades de la vida política, la adecuación a las necesidades de la guerra, y la belleza. A este propósito señala Newman ${ }^{22}$ que los fundadores de ciudades en la Grecia antigua parecen haber prestado más atención a los dos últimos puntos que a los primeros, aduciendo al respecto la autoridad de Estrabón ${ }^{23}$. En la obra del geógrafo de Apamea encontramos, como era de esperar, una realidad compleja. Las condiciones naturales de un país desde la perspectiva de la defensa son objeto de la previsible atención, mientras que en la parte dedicada a Roma ${ }^{24}$ se subraya, en cambio, cómo la carencia de defensa ha sido obviada mediante la actuación de los hombres, en particular de los hombres públicos más eminentes ${ }^{25}$.

dos, como pudo reconocer Gernet a propósito de un buen número de relatos utópicos. L. Gernet, "La ciudad futura y el país de los muertos", en Antropologia de la Grecia antigua, Madrid 1980, pp. 123-35 (publicado en REG 46, 1933, pp. 293-310).

${ }^{20}$ Utopias of the Classical World, Londres 1975, p. 124.

${ }^{21}$ Pol. 1330 a-b. En $R .1359$ b el estagirita habia anotado el tema de la defensa del territorio entre los propios de la oratoria deliberativa.

2. The Politics of Aristotle, III, Oxford 1902, p. 396.

${ }^{23}$ Cf. Str., V 3,8.

${ }^{24}$ Str., V 3,7.

${ }^{25}$ Resulta curioso a este respecto que, cuando en Estrabón encontramos la presentación de un país cuasi o parautópico el de los Albaneses (en XI 4), territorio no remoto entre los Iberos y el Mar Caspio-, se hace mención de sus defensas naturales dentro de lo que permite el respeto a la realidad geográfica tal como Estrabón la veía, y sus habitantes son caracterizados, en estrecha relación con la parte

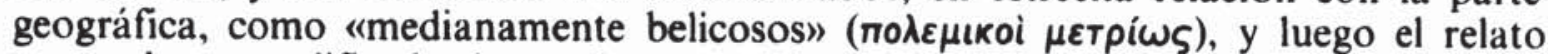

que hemos calificado de cuasi o parautópico -- insiste en la prodigiosa feracidad natural del país, si bien omite aspectos tan característicos de la narración utópica como el pacifismo o las relaciones sexuales libres. 
En la apócrifa Carta de Aristeas a Filócrates podemos leer la descripción de un territorio que obedece a una "tópica" semejante ${ }^{26}$. La descripción de Palestina que completa la de Jerusalén y su Templo presenta con nitidez los motivos de la feracidad del territorio y de la privi-

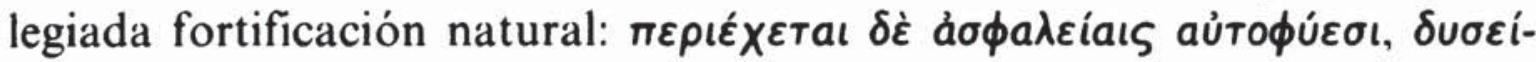

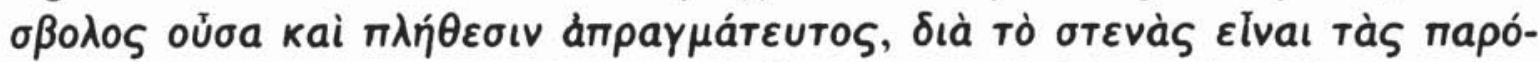

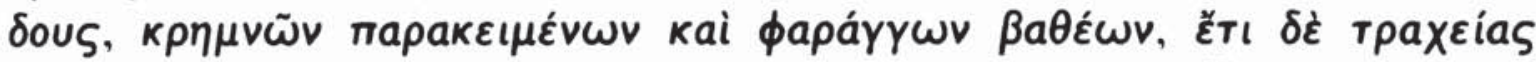

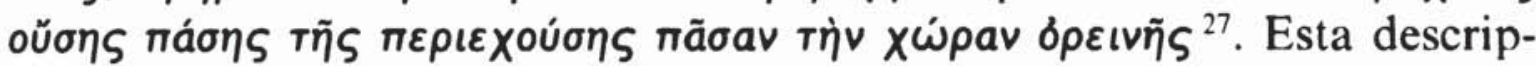
ción se articula en torno a una synkrisis entre Palestina y Egipto que aparece explícita en las referencias a Alejandría (109-111), capital de Egipto cuyas dificultades, derivadas de su propia prosperidad, ha sabido evitar Jerusalén, y al río Nilo (116), responsable de la fertilidad del país y equiparado en este punto al Jordán. La referencia a la fortificación natural del país se explica por una doble ascendencia igualmente retórica: el de los esquemas tópicos del encomio y la especial fortuna que ha tenido este tópico en concreto en la extensa tradición griega sobre el país del Nilo.

3. Se hace preciso reconocer la raigambre retórica de los temas que centran la descripción diodorea de Egipto en I 30-31: la feracidad de la tierra ${ }^{28}$ y la polyanthrōpia que provoca ${ }^{29}$, así como la seguridad de las defensas naturales ${ }^{30}$. Pero ocurre que el énfasis que en I 30-31 se pone incluso en cuanto a extensión de texto en el carácter formidable de las defensas naturales de Egipto parece haber oscurecido el hecho de que en dichos capítulos se encuentren los demás elementos habituales de la exaltación del país. Reconocidos los rasgos utópicos de I 30-31 en sus términos precisos de topoi literarios tradicionales, cabe replantearse la dificultad - realmente grave que presentaba la hipótesis de que

Por otra parte, en el Critias platónico se insiste en que en la Atenas primitiva (cuyas defensas naturales eran, por supuesto, tan mediocres como las de la Atenas histórica) se atendia cuidadosamente al mantenimiento de un ejército capaz de asegurar la defensa (112 d).

26 Incluso con la mención de la entrada al país de bienes procedentes de otros territorios.

27 Aristeas Iudaeus, 118.

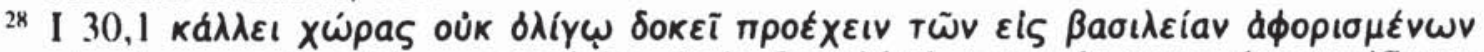
rón $\omega v$ ha de ser entendido a propósito de la feracidad, como lo pone de manifiesto I 34,2 acerca de una parte de Egipto, donde el sentido 'feraz' no ofrece duda. Pero es, además, posible que en este pasaje se contenga una mención sucinta de la belleza del territorio, topos frecuente en este tipo de descripciones, como hemos visto. No hay duda, como luego veremos, del carácter deliberadamente sintético de las partes de I 30-31 en que aparecen los componentes del esquema distintos del de la fortificación natural.

29 I $31,6-8$.

30 I $30,1-31,6$. 
Diodoro hubiera tomado dichos capítulos de Hecateo y el resto de la exposición geográfica (I 32-41) de una fuente distinta (Agatárquides, bien de modo directo, bien a través de algún autor intermedio). Es mucho más económica, por supuesto, la hipótesis de que Diodoro haya tomado de una única fuente el conjunto de la parte geográfica del libro I y que él mismo haya organizado los capítulos 30-31 en torno al esquema retórico tradicional. La suposición de que sea el propio Diodoro el autor de estos párrafos, que podría parecer inadmisible a quienes atribuyen al siciliano un modo de actuación exclusivamente mecanicista, es reforzada por el supuesto de que el historiador haya procedido sobre la base de un esquema retórico tradicional que en su época, además, se había consolidado precisamente en relación con Egipto.

En efecto, el esquema al que nos venimos refiriendo lo encontramos aplicado a Egipto en el Busiris de Isócrates, que en buena parte es un ह̌naıvos de Egipto (ejemplo particularmente tomprano entre los conocidos $\left.{ }^{31}\right)$, hasta el punto de que en $\S 30$ Isócrates ha de hacer frente a una eventual objeción de que se está dejando a un lado el auténtico propósito del discurso: elogiar a Busiris. Este mismo capítulo 30 es muy relevante porque en él Isócrates resume su encomio de Egipto con las si-

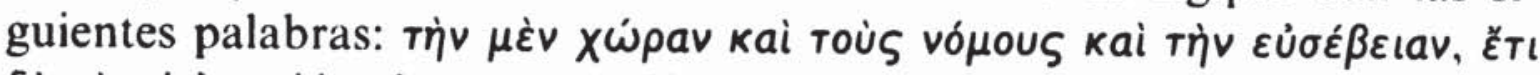

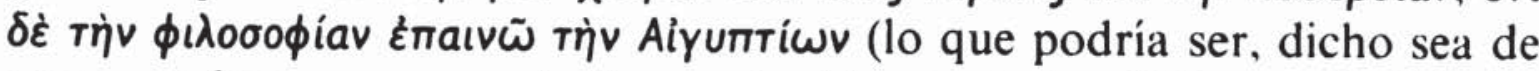
paso, un buen resumen de los contenidos de todo el libro I de Diodoro). Así pues, un capítulo específico del elogio lo constituye el del país, de cuyas cualidades se da un primer resumen sucinto al comienzo del mismo ${ }^{32}$ : excelente localización, feracidad y productividad extraordinaria y variada, y fortificación natural proporcionada por el Nilo (con la eficaz expresión "amurallada por la inmortal muralla del Nilo»). Resulta ilustrativo constatar que Froidefond, quien, como indica el título de su obra, detiene su indagación mucho antes del tiempo de Diodoro, percibe en estos pasajes isocráticos un enriquecimiento de la "tópica» tradicional relativa al Nilo. El autor ateniense, al igual que probablemente sus predecesores, deduce la lección de las expediciones de Egipto, que habían demostrado ampliamente el valor estratégico del Delta. Las consideraciones del Busiris, pese a su carácter general, no dejan de fun-

3 Podemos sin embargo rastrear los tópicos ya en Pi., fr. 82 Snell-Maehler ràv

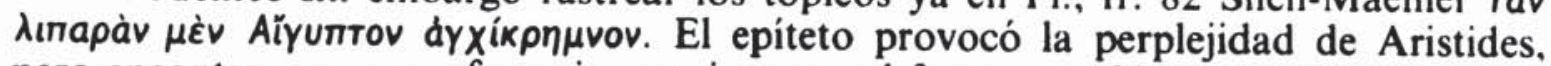
pero encontramos una referencia semejante en el fragmento 201 Snell-Maehler, en el

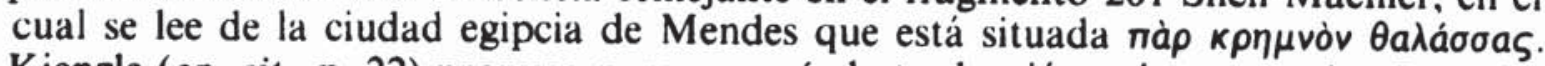
Kienzle (op. cit., p. 22) propuso para $\kappa \rho \eta \mu v o ́ v$ la traducción más neutra de "Strand". Sobre la inaccesibilidad de Egipto en Homero, cf. Ch. Froidefond, Le Mirage Égyptien dans la littérature grecque d'Homère d̀ Aristote, Aix-en-Provence 1971, pp. 20-1.

${ }^{32}$ Cf. Busiris 12-13. 
darse en acontecimientos muy precisos: se trata de los desastres, aún muy recientes en 385 a.C., de los generales de Artajerjes o, más en concreto aún, de la primera estancia de Cabrias en Egipto, estancia que el general ateniense aprovechó con toda probabilidad para comenzar los trabajos de fortificación del Delta, su mayor título de gloria ${ }^{33}$. Es incontestable que el tema del Nilo, hasta entonces poético o científico, se encontraba enriquecido por Isócrates con consideraciones económicas y políticas en la obra que mencionamos. Delatte ${ }^{34}$ había ido más lejos y leido sus $\$ \S 11$ ss. como una expresión de la vocación insular y autárquica de Egipto y como demostración del modo en que ha podido constituirse el "orden» social sobre el que reposa la "civilización» egipcia. Tal lectura, que no parece provocar el asentimiento de Froidefond ${ }^{35}$, resulta particularmente interesante por mostrar que una interpretación similar a la que Murray extrae de los pasajes que consideramos del libro I diodoreo parece obtenerse también a partir del estudio de los $\$ \$ 11$ ss. del Busiris isocrático mediante una lectura igualmente sesgada en el sentido de "inaccesibilidad" de la noción de "inexpugnabilidad", que es la realmente central y presente tanto en el texto de Isócrates como en el de Diodoro ${ }^{36}$.

El doble motivo constatable en el Busiris isocrático de la protección y la abundancia que el Nilo proporciona a Egipto es justo el que abre la exposición de la geografia egipcia de Diodoro, si bien lo que en Isócrates se reduce al Nilo ha dado paso en el siciliano a un cuadro imponente de fortificación natural en el que se han aprovechado los datos de toda una tradición geográfica sobre Egipto. Coincide también Diodoro con Isócrates en la presentación de los múltiples canales de Egipto como un medio de distribución de bienes que permite a todo el país gozar de la mayor prosperidad. Diodoro añade a estas consideraciones otras de carácter estrictamente estratégico cuyo rendimiento será notable en la exposición de los acontecimientos históricos.

Pero el paralelo más fiel a la presentación diodorea de los límites de Egipto lo encontramos, curiosamente, en un autor contemporáneo y de ambiciones igualmente amplias. Estrabón, en efecto, cierra su descripción de Egipto con los términos siguientes:

${ }^{33}$ Froidefond, op. cit., pp. 250-1.

34 Essai sur la politique pythagoricienne, Lieja 1924, p. 94.

${ }^{35}$ Op. cit., p. 238 y n. 17.

36 En efecto, en Busiris 13 se subraya que el Nilo es inexpugnable y dificil para los atacantes, pero favorable para el comercio, y a continuación (ibidem 14) se reitera la buena disposición de Egipto para el comercio, tanto de exportación como de importación. 


\begin{abstract}
Egipto fue básicamente pacifico desde el principio, tanto por la autosuficiencia del pais como por ser dificilmente atacable para los de fuera: por el norte está fortificado por una costa sin puertos y por el mar egipcio, a este y oeste por montes desiertos, los de Arabia y Libia, como dijimos; y, en fin, al sur están los Trogloditas, los Blemios, los Nubios y los Megabaros, razas etiopes que habitan más arriba de Siene, gentes nómadas que no son muchos ni belicosos (...). $\mathrm{Y}$ también ahora el país entero mantiene el mismo carácter pacífico. Véase la prueba: una guarnición de tan sólo tres cohortes, y ni siquiera completas, es suficiente para que los Romanos guarden el pais ${ }^{37}$.
\end{abstract}

Estrabón adopta el punto de vista de la administración y el gobierno romano sobre una provincia, con preocupaciones bien características como la defensa de las fronteras y el mantenimiento del orden interno. Es este interés administrativo el que confiere relevancia al carácter pacifico de la nación y a sus causas, así como a la facilitad que el país puede ofrecer a la hora de mantener a los bárbaros fuera de los límites del imperio ${ }^{38}$.

Pero el estrecho paralelismo entre el Egipto de Diodoro y el de Estrabón -- dos autores prácticamente contemporáneos entre los que nadie ha postulado dependencia alguna - invita sobre todo a ciertas reservas al afrontar el problema de las fuentes. La coincidencia indica más bien la difusión de un motivo que, como lugar común de la descripción del país del Nilo, permite su utilización y adaptación a diversos intereses.

Una prueba del carácter de tópico laudatorio que para los antiguos tenía la alabanza de las defensas de Egipto nos la brinda el comentario de Eustacio a la descripción que hace de Egipto Dionisio el Periegeta. En los versos 239 ss. trata éste la forma y los límites del país del Nilo por medio de la tradicional referencia al triángulo que forman la costa y los dos lados que se juntan en Siene. Eustacio, al comentar estos versos que entiende - como toda la descripción de Egipto - en términos

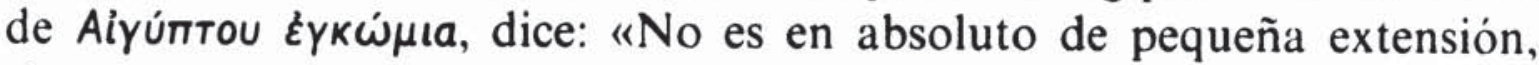
sino que supera a las demás por sus pastos, por sus prados, así como por producir todo lo espléndido. Otros cuentan que está fortificada naturalmente y dicen que es inatacable por tierra y que carece de puertos en la zona marítima" ${ }^{39}$. Eustacio ha completado la "tópica» fundamental del elogio de Egipto, que en Dionisio aparecía incompleta ${ }^{40}$, con la

${ }^{37}$ Str., XVII 1,53; cf. también XVII 1,19 y 21, donde se menciona el dificil acceso por el Pelusio, rodeado de pantanos y marismas.

${ }_{38}$ Cf. P. Thollard, Barbarie et civilisation chez Strabon, Paris 1937, p. 82.

39 GGM II, 259.

$40 \mathrm{Tal}$ vez haya interpretado en este sentido el verso 245 . El carácter retórico de los encomios geográficos así como la interpretación de Eustacio ha sido señalado 
referencia a otro autor (Estrabón con toda probabilidad) en el que el tópico aparecia adecuadamente tratado. Interesado en una presentación más contemplativa del mundo, Dionisio habría potenciado, a la inversa de Diodoro, los tópicos referentes a la feracidad de la tierra; Eustacio equilibra el conjunto de acuerdo con sus fines exegéticos y didácticos.

4. Es el momento de preguntarse cuáles son los intereses de nuestro autor en el aprovechamiento de esta tradición científica y literaria sobre el país del Nilo. Hemos tenido ocasión de apuntar que lo característico del historiador de Agirio es la hipertrofia del tópico de las defensas naturales de Egipto desde el punto de vista de su «inatacabilidad» (y no de su inaccesibilidad, pues el acceso era en principio posible). Ahora bien, las razones de este desarrollo, como veremos inmediatamente, no pueden ser imputables sino a los peculiares intereses de Diodoro en la redacción de su monumental Biblioteca Histórica. Para ello analizaremos el pasaje en cuestión en el contexto del libro I y del conjunto de la obra.

El privilegio de Egipto es ciertamente inseparable de su condición geográfica. Egipto, sin embargo, ofrece algo más aparte de las ventajas de su suelo y de su régimen fluvial. Al comenzar la sección dedicada a la geografia, Diodoro afirma que este país aventaja a las demás tierras que conforman un reino por su fortificación natural:

\footnotetext{
Egipto se extiende más bien hacia el mediodia. Por sus defensas naturales y la excelencia del territorio parece que supera en mucho a otras regiones que conforman un reino. Al oeste, en efecto, está defendido por la parte desierta y salvaje de Libia, una enorme extensión que por falta de agua y escasez de todo tipo de alimento presenta una travesia no sólo penosa, sino también altamente peligrosa. Al sur lo defienden las cataratas del Nilo y las montañas que las circundan, pues desde la región de los Trogoditas y las zonas extremas de Etiopía en una distancia de cinco mil quinientos estadios no es fácil navegar por el río ni hacer la ruta a pie, a menos que se disponga de un equipamiento regio $o$, en todo caso, bien considerable. En cuanto a las zonas que dan a oriente, unas las defiende el río, otras las rodea el desierto y unas llanuras pantanosas llamadas "Baratra» (...). El cuarto lado, bañado por aguas que apenas ofrecen un puerto, tiene ante si como defensa el mar de Egipto, que presenta una travesía muy dura y un acceso a tierra especialmente dificultoso (I 30-31,1 $)^{4 !}$.
}

por Ch. Jacob en "L'oeil et la mémoire: sur la Périégèse de la Terre habitée de Denys», en Jacob y Lestringant, op. cit., p. 52.

${ }^{41}$ Este diseño cuadrangular de Egipto encuentra paralelo, aparte del pasaje de Estrabón ya citado, en un fragmento del epitome de Agatárquides realizado por Focio (444b39-40 Henry): "Cuatro son las zonas que encierran a Egipto: el mar al norte, desiertos por el este y el oeste y Etiopia al surn. En cuanto a la costa que limita Egipto por el norte, Diodoro da tres medidas. La primera es de 5.000 estadios desde Paretonio hasta Jope (Jafa) en Celesiria, en la que sólo se puede encontrar, se- 
Ahora bien, parece que lo que gobierna esta topografia de Egipto es un interés práctico que coincide bastante con lo que, a propósito de Polibio, Pédech llamaba "primière manière» de la geografia ${ }^{42}$. Egipto aparece dibujado desde un punto de vista predominantemente "hodográfi$\operatorname{con}^{4}{ }^{43}$ : los accesos y travesías con la indicación de las distancias, los obstáculos y los peligros que en cada caso se encuentran. Más en concreto, todo parece orientado al interés del militar y el estratega, como se pone de manifiesto en la descripción de los pantanos llamados "Baratra»: ejércitos enteros han desaparecido en sus arenas por la inexperiencia de sus generales. Los libros sucesivos de la Biblioteca demostrarán cuán estrecho es el lazo que une esta descripción a los acontecimientos de la historia política y militar de Egipto. Por de pronto, queremos poner de manifiesto que estos capítulos no son sino la expresión más plena de un tópico recurrente en el libro I.

Para ello es conveniente situar a Diodoro en la historia de un problema tan tradicional de la geografia griega como es el de los límites de Egipto. Para la incipiente investigación geográfica, Egipto se convierte, al menos desde Hecateo, en una pieza fundamental con la muy ambigua función de dividir las masas continentales ${ }^{44}$. Cuando Heródoto intenta, a partir de esta tradición, presentar una imagen consistente de Egipto,

gún Diodoro, el puerto de Faro (I 31,1). Según J. Stern (Greek and Latin authors on Jew:s and Judaism, I, Jerusalén 1976, fr. 56 [= D.S., I 31,2], pp. 169-70), Jope era el puerto principal de Judea en la época de Diodoro, aunque la ciudad tuvo cierta importancia en la historia de los Diádocos (D.S., XIX 59,2; 93,7; I., AI XIV 4,4; 10,6; XV 7,3; XVII 11,4. La Carta de Aristeas la da como uno de los principales puertos de Palestina). Pero la razón principal para que Jope sea un punto de referencia para la costa de Egipto nos la da Estrabón (XVI 2,28): en Jope la costa que partía de Egipto, que hasta el momento se extiende en dirección este, gira de manera significativa hacia el norte. Paretonio aparece ya en el Periplo de Pseudo-Escílax (\$106) como limite costero occidental de Egipto. De aquí partia la ruta hacia el oasis de Siwa (cf. Str., XVII 1,14). En I 31,6 da Diodoro una medición global de Egipto: 2.000 estadios de costa y 6.000 hacia el interior. En I 34,1 ofrece las distancias para el Delta: 1.300 estadios en la costa por 750 estadios para los lados que suben al interior, pero en 57,4 la distancia de Pelusio a Heliópolis, esto es, uno de los lados del Delta, es de 1.500 estadios, medida que coincide con la que da Heródoto en II 7. Por otro lado, 1.300 estadios de costa es lo que dan Estrabón (XVII 1,6), Plinio (NH V 19) más o menos y Pseudo-Escílax, si aceptamos la corrección de Müller (cf. $G G M$ I 81 ), corrección que, sin embargo, fue rechazada por Jacoby; cf. $R E$ 14. 1912, col. 2679; en contra, Peretti, op. cit., p. 277.

42 La méthode historique de Polybe, París 1964, p. 529 ss.

${ }^{43}$ Sobre este concepto fundamental para la comprensión de la geografia antigua vid. P. Janni, La mappa e il periplo, Roma 1984.

44 F. Gisinger, s.u. "Geographie», RE Suppl. IV, 1924, cc. 552-6. Sobre la atracción que Egipto ejerció sobre el mundo jonio véase Froidefond, op. cit., p. 118 ss.; sobre Heródoto heredero de Hecateo, vid. T. S. Brown, «Herodotus speculates about Egypt», AJPh 86, 1965, pp. 60-76. 
se debe enfrentar al problema de que este país, en tanto que límite él mismo, carece a su vez de límites. La solución de Heródoto, como se sabe, responde plenamente a sus intereses históricos: Egipto es la tierra habitada por los egipcios, como Asiria la de los asirios, y son egipcios quienes beben el agua del Nilo ${ }^{45}$; se trata de un país con sus habitantes y fronteras, definido por sus usos e instituciones, conquistado y conquistable. En el libro I de Diodoro el problema ya no es tal, pues el punto de vista no es el de la geografía especulativa, sino el mucho más concreto de la topografia o corografía. Esto explica la ausencia casi completa de alusiones a la naturaleza aluvial del país del Nilo, que aparecerá, sin embargo, en la descripción de Etiopía del libro III como prueba del carácter reciente de la tierra de Egipto ${ }^{46}$.

El desplazamiento de intereses a partir de esencialmente los mismos datos es significativo. Los elementos que para autores como Heródoto constituian un quebradero de cabeza que amenazaba con reducir Egipto a un sinsentido geográfico e histórico - los desiertos de Libia y Arabia que se ciernen sobre el valle del Nilo hasta reducirlo al mínimo, la ambigua línea de la costa en constante formación, son para Diodoro formidables murallas defensivas que abarcan un reino privilegiado por el hecho de encontrar una delimitación natural. No todos los grandes reinos tienen tanta suerte. Así, por ejemplo, los escitas hacen su entrada en Diodoro como un pueblo insignificante, vagamente localizado en el curso del Araxes, hasta que por obra de un monarca se constituyen en un reino cuyos limites están definidos por accidentes naturales bien notorios; sólo así entran en la historia ${ }^{47}$. Las fronteras son, sobre todo, condición de la existencia misma de un reino.

Fuera ya de la sección geográfica, es interesante poner de relieve la continuidad de los intereses estratégicos en otros momentos del libro I. Así, por ejemplo, en el caso de la ciudad de Menfis, la reelaboración de las noticias geográficas tradicionales desde el nuevo punto de vista es todavía más llamativa. Cercada por el agua del Nilo por todos lados excepto por uno, en el que queda protegida por un promontorio artificial en el que se levanta una ciudadela imagen invertida, por tanto,

${ }^{45}$ Heródoto, II 17,1 y 18. Definición que, por otro lado, responde bien a la concepción que los egipcios mismos tenían de su tierra; vid. A. B. Lloyd, Herodotus. Book II. Commentary 1-98, Leiden 1976, p. 90.

46 D.S., III 3,2-3.

47 D.S., II 53. Análogamente, la labor de los primeros reyes de Asiria es la de delimitar la geografia de su imperio con un recorrido por los limites de Asia; cf. D.S., II 3 ss. 
de la fortificación natural del país-, Menfis sobresale por su acierto estratégico ${ }^{48}$.

El cambio de óptica puede apreciarse si se recuerda la importancia que en la geografia jonia tenía la ciudad de Menfis como punto de referencia para la descripción de Egipto. Hecateo de Mileto veía en esta ciudad el punto máximo de estrechamiento del país y el comienzo de una extensión progresiva que da a la tierra de Egipto la forma de «doble hacha" ${ }^{49}$. Heródoto parece polemizar con esta concepción cuando presenta su problemática visión del valle del Nilo ${ }^{50}$ : el estrechamiento de Egipto comenzaba, según este autor, en Heliópolis y continuaba en una distancia de cuatro días río arriba ${ }^{51}$.

Para Diodoro, en cambio, la ciudad de Menfis es ante todo un eje histórico es la sucesión que va desde Tebas a Alejandría. De ella destaca, en primer lugar, su oportuna localización como llave de la navegación río arriba; por otro, su imponente fortificación, resultado de las obras del faraón Ucoreo. Esta condición estratégica, natural y artificial al mismo tiempo, atrae a los faraones siguientes, que abandonan la monumental Tebas para establecerse en la nueva capital, en la que habrán de residir hasta que Alejandro el Grande funde la ciudad que lleva su nombre. Es curioso constatar que en la fundación de Alejandría tienen también un lugar relevante las consideraciones defensivas ${ }^{52}$.

48 D.S., I 50,3-6.

49 Esta imagen aparece en el Periplo de Pseudo-Escílax 105 (GGM I, pp. 80-1) y fue atribuida por Jacoby a Hecateo en FGrHist I Comm., p. 368 (a propósito de 1 F 301) y s.u. "Hekataios», $R E$ 14, 1912, col. 2680. En contra, A. Peretti, Il Periplo di Scilace. Studi sul primo portolano del Mediterraneo, Pisa 1979, p. 384 s. Para Peretti este pasaje es, en primer lugar, un añadido posterior al texto primitivo, como denuncia su carácter especulativo "di tavolino", ajeno a los intereses del Periplo original, estrictamente prácticos y concentrados en la línea de la costa. Tampoco es remontable a Hecateo, pues supone un conocimiento del territorio más arriba del Delta: la otra hoja del hacha estaría constituida por la depresión del Fayum, a la que Heródoto (II 149) atribuye un perímetro igual al de la costa de Egipto. A propósito de la forma de Egipto, es característico de Diodoro el abandono de una imagen tradicional para la descripción de este país (de la que deriva, en definitiva, por extensión analógica la del "doble hacha»). Nos referimos a la figura triangular que subyace en la representación de Egipto desde Esquilo hasta Dionisio Periegeta; cf. J.-P. Bertrand, «De l'emploi des métaphores descriptives par les géographes de l'Antiqui-

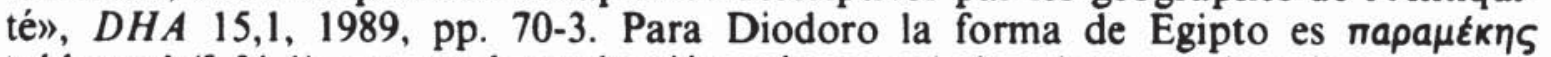
'oblonga' (I 31,1), aunque la traducción más apropiada tal vez sea la más general de "alargada». También Estrabón en un problemático pasaje describe la tierra de Egipto como un "vendaje».

so Lloyd, op. cit., pp. 54-7.

51 Heródoto, II 8. Sin una anulación completa del carácter axial de Menfis en el diseño de la geografia egipcia, cf. II 10,$1 ; 12,1 ; 99,4 ; 150,1 ; 158,2$. De especial interés es II 14,2, incompatible, como reconoce el mismo Lloyd (op. cit., p. 74), con las afirmaciones de Heródoto en II 5. Véase también II 13,2.

52 XVII 52,2 y 3. Diodoro hace de nuevo alusión a su visita a Egipto. 
Todavía dentro de la historia egipcia no faltan otras referencias a la fortificación que hacen de este tema un auténtico tópico ${ }^{53}$. Destacaremos aquí la que creemos más significativa. Entre las obras por las que destaca la mítica figura de Sesostris se encuentra, en primer lugar, la construcción de una muralla defensiva en el ya de por sí inexpugnable desierto de Siria. Pero su labor más significativa a este respecto fue el drenaje de Egipto por medio de una densa red de canales, cuyo efecto, según Diodoro, aparte de garantizar una comunicación de bienes entre todas las partes de Egipto, fue hacer especialmente dificil una eventual invasión ${ }^{54}$. La noticia, como se sabe, remonta a Heródoto, pero para este autor la operación del faraón tiene un efecto diferente, sin referencia alguna a ventajas estratégicas ${ }^{55}$. En Heródoto, gracias a estas obras, el territorio de Egipto adquiere dimensiones reales, unos límites de los que carecía cuando era practicable a caballos y carros. La acción regia configura el país como un espacio sobre el que se ejerce un poder que lo delimita ${ }^{56}$. Ahora bien, para Diodoro esto es algo que a Egipto le viene dado de forma natural, por lo que la labor que el rey asume es la de perfeccionar en su debida orientación aquello que la naturaleza ofrece espontáneamente. Impone así un modelo a los futuros gobernantes de Egipto, que de manera constante se encargarán de reforzar los límites del pais ante las múltiples amenazas de invasión.

5. Para la correcta valoración de la opción diodorea al potenciar de entre los tópicos del encomio la imagen "poliorcética» de Egipto, imagen tan marcadamente plasmada en el libro I, es fundamental reco-

${ }^{33}$ Debe añadirse la explicación que da Diodoro del culto al cocodrilo: su abundancia hace del río una barrera infranqueable frente a las invasiones provenientes de Arabia y Libia (I 89,2). En la misma sección sobre los animales sagrados, Diodoro cuenta que una vez los lobos se concentraron en Elefantina y defendieron Egipto de una invasión procedente de Etiopia, por lo que recibieron honores divinos (I 88,7).

54 D.S., I 57,2-3.

$5 s$ Heródoto (II 108,2-4) atribuye a Sesostris razones de salubridad. En el reciente libro de C. Obsomer (Les campagnes de Sésostris dans Hérodote. Bruselas 1989) podemos encontrar una detallada comparación de las fuentes antiguas sobre las campañas de este faraón, entre las cuales la de Diodoro reviste un notable interés. Este aspecto de la versión diodorea de las empresas hidráulicas de Sesostris es apenas aludido (p. 38), aunque creemos que es revelador de la orientación que Diodoro impone a la historia egipcia.

56 Sobre estos aspectos, vid. F. Hartog, Le miroir d'Hérodote. Essai sur la représentation de l'autre, París 1980 , pp. 36-40. O. Longo ("Idrografia erodotea", QS 24 , 1986, pp. 29-30) ha criticado la interpretación de Hartog, construida, según él, sobre una excesiva asimilación de Egipto a Escitia. No obstante, a propósito de las obras hidráulicas de Sesostris, Longo sobreentiende una valoración defensiva, inexistente en Egipto, a partir de una comparación con Babilonia, donde tal motivación aparece explícitamente formulada. 
nocer la continuidad de la misma en el resto de la Biblioteca. Esto nos permite concluir que Diodoro, al margen de la fuente seguida en los capítulos geográficos, ha sabido integrar esta imagen en la narración de los acontecimientos y sacar rentabilidad de su peculiar orientación estratégica.

En XV 42 ss. narra Diodoro el fracaso de la expedición enviada por Artajerjes en el 374 a.C. para reconquistar Egipto:

El rey de los egipcios Nectanebis conocía el tamaño del contingente persa, pero confiaba sobre todo en la fortaleza del territorio, pues Egipto es completamente inatacable. Además, estaban bien fortificados todos los posibles accesos, tanto por tierra como por mar. En efecto, el Nilo vierte al mar sus aguas por siete bocas, en cada una de las cuales habia edificada una ciudad con grandes torres a cada lado del cauce, y un pontón de madera dominaba la entrada río arri$\mathrm{ba}^{57}$. Pero sobre todo acondicionó la boca Pelusia por ser la primera que sale al paso a los que vienen de Siria, en la opinión de que los enemigos intentarian el ataque sobre todo por este sitio.

Ante la formidable fortificación del lugar, la expedición se dirige a otra boca que consiguen tomar. Pero a continuación surge la discordia entre los mandos:

Ificrates, informado por los prisioneros de que Menfis estaba desguarnecida, siendo como era la ciudad estratégicamente mejor situada de las de Egipto, era del parecer de que habia que remontar al punto el rio hasta Menfis, antes de que se presentaran las tropas egipcias $(43,1)$.

El retraso en la decisión da tiempo a lo egipcios para rehacerse, enviar tropas a Menfis y emprender la reconquista de la ciudadela tomada por las tropas persas. Durante el asedio de la misma el río Nilo colabora de manera inesperada:

Como se prolongaba el asedio a esta plaza y los etesios ya habian comenzado a soplar, el Nilo, ocupando en su crecida todo el lugar con su imponente caudal, iba fortificando cada vez más Egipto $(42,4)$.

De manera semejante, la expedición enviada por Artajerjes Oco en el 350 a.C., que culminará con la conquista del país, tropieza con lo que para Diodoro era la más impresionante fortificación natural de Egipto:

37 Diodoro menciona en I 33,8 , esto es, la parte "agatarquidea", las fortificaciones existentes en cada desembocadura del Nilo en términos semejantes; se trata de un elemento más de cohesión entre las dos partes en que se suele dividir la sección geográfica. 
Al llegar al gran lago en el que se encuentran los lugares llamados Baratra perdió una parte de su ejército por el desconocimiento del terreno. Sobre la naturaleza de este lago y el caso peculiar que en él se da ya hemos hablado previamente en el libro primero y, por tanto, evitaremos decir dos veces lo mismo (XVI 46,4-5).

La referencia es obviamente a I 30,4, pero, sin duda, la extensa descripción que Diodoro hace de las arenas movedizas en 30,4-9 responde a la rentabilidad que el fenómeno tiene en los acontecimientos históricos. Las operaciones de fortificación de los egipcios ante la noticia de la invasión persa se concentran de nuevo en la boca de Pelusio $(45,6)$. También Nectanebo ha guarnecido con fortines toda la orilla del Nilo que da a Arabia (47,6-7).

Significativa asimismo es para nosotros la narración de la fallida invasión de Egipto emprendida por Perdicas, en la que Ptolomeo confirma con la lanza el derecho sobre su futuro reino. Tras fortificar convenientemente los lugares estratégicos (XVIII 33,3), resiste con éxito el ataque del ejército de Perdicas contra uno de los baluartes en la ribera del Nilo. La narración de la maniobra de Perdicas en la zona de Menfis demuestra una vez más el carácter fundamental de esta ciudad en la estrategia de conquista:

Tras emprender la marcha por la noche llegó frente a Menfis, donde el Nilo se divide y forma una isla que puede dar cabida con seguridad al más grande campamento o ejército. Pues bien, la travesia a esta isla se llevaba a cabo penosamente por la profundidad del Nilo: la corriente del rio les llegaba a la barbilla y hacia perder el equilibrio a los que lo atravesaban, que se veian estorbados por el peso de las armas (34.6-7).

Perdicas intenta resolver esta dificultad por el procedimiento de frenar la corriente con los elefantes. No obstante,

ocurrió algo inusitado en la travesia del ejéreito: cuando los primeros ya habian atravesado el río, a los que iban a continuación les sobrevino un gran peligro, pues el rio, sin causa aparente alguna, se fue haciendo cada vez más profundo y. conforme los hombres se hundian, se iban viendo todos en grandes apuros. Cuando se investigo la causa de esta subida era imposible dar con la verdad: unos decian que rio arriba se habia abierto un canal que antes estaba cerrado por un dique, y que, al juntarse el agua con el caudal del rio, lo hizo más profundo; otros, que habian tenido lugar en las tierras altas unas lluvias que aumentaron el volumen del Nilo $(35,2-4)$.

Diodoro aduce a continuación la que él cree verdadera causa de tan curioso fenómeno; lo importante es, con todo, que esta narración juega 
con los elementos de la sección geográfica egipcia: fortificación gracias a los elementos naturales e incluso una pequeña doxografia para una crecida en miniatura, en la que tiene cabida una explicación que es, a escala reducida, la que Agatárquides propone para la crecida del Nilo, aunque ahora Diodoro no le presta crédito. Añádase que la mayoría de los hombres arrastrados por la corriente fueron, según Diodoro, devorados por las "fieras del Nilo" $(35,6)$, las cuales, como nos dice el mismo Diodoro en el libro I, constituyen, en efecto, un factor no despreciable de la fortificación natural del país ${ }^{58}$.

Finalmente, en el último de los libros de la Biblioteca transmitidos de manera no fragmentaria, dentro del relato del intento de invasión por parte de Antígono en el 306, Diodoro hace de nuevo mención recurrente a las dificultades naturales que presenta el pais: las llanuras pantanosas llamadas «Baratra» (XX 73,3), el dificil acceso por la costa (74, $3)^{59}$ y el río como muralla $(76,4)$. Antígono plantea, en fin, la posibilidad de invadir el país cuando el río esté más bajo $(76,5)$.

En resumen, para Diodoro la "defendibilidad» de Egipto constituye un motivo fundamental de la descripción del país, no sólo en el logos egipcio, sino también, como hemos tenido ocasión de constatar, en otros pasajes de la Biblioteca en los que Egipto cobra protagonismo. Ahora bien, el rendimiento del motivo es ahora esencialmente historiográfico en el sentido más estricto de la palabra: historia política y militar, pragmática, por hablar en términos polibianos. Y en esta uniformidad es difícil dejar de ver una elaboración del propio Diodoro, interpretable dentro del conjunto de ideas que articulan la Biblioteca.

En un artículo reciente insistía Sartori ${ }^{61}$ en la profunda uniformidad de orientación y temas que encontramos en los cinco primeros libros de la Biblioteca. Especialmente interesante para nosotros es la observación de Sartori acerca de la dimensión histórica que impregna el abigarrado material que Diodoro nos of rece en sus primeros libros. En ellos se puede detectar la presencia de una concepción global de la evolución del hombre desde un estado animal, evolución en la que se hace imprescindible la figura del monarca inventor y benefactor cuya acción supone un salto cualitativo en el progreso hacia un estado civilizado. El estado natural y sus recursos, las fuerzas que empujan y permiten al hombre avanzar (utilidad, provecho, experiencia, necesidad), pero sin apenas

${ }^{58}$ D.S., I $89,2$.

59 Cf. D.S., XX 76,2: «Demetrio, tras fracasar este desembarco y oír que toda la costa adyacente estaba fortificada de manera natural por pantanos y marismas...".

60 "Storia', 'utopia' e mito nei primi libri della Biblioteca Storica di Diodoro", Athenaeum 62, 1984, pp. 491-536, esp. 511-16. 
traspasar el umbral de la animalidad hacia el estado más primitivo de civilización. Es la figura del monarca la que asume el cometido de hacer avanzar la sociedad, a través de la institución del estado y la fundación de la ciudad, hacia cotas de civilización aceptables. De hecho, pese a las formidables murallas naturales una parte importante de Egipto es todavía accesible (عüßaтov) a caballos y carros, hasta que las obras emprendidas por el faraón Sesostris alteran radicalmente su naturaleza ${ }^{61}$.

El motivo de la fortificación natural de Egipto responde perfectamente a este esquema, en la medida en que aquello que la tierra brinda de modo natural debe ser promocionado y perfeccionado por la acción de los monarcas, quienes en definitiva son los garantes de la seguridad del país. Si este planteamiento aparecía ya en Hecateo es cosa indemostrable, a no ser por una referencia viciosa al mismo Diodoro. En cualquier caso, hay que reconocer al escritor de Agirio la capacidad de dar coherencia a su Biblioteca por medio del aprovechamiento de motivos especialmente relevantes desde el punto de vista de la historia pragmática.

\author{
JESUIS LENS TUERO \\ Javil: Campos Darocia
}

"No existe contradicción alguna entre la presentación "poliorcética" de Egipto en la sección geográfica y esta afirmación de Diodoro acerca de la accesibilidad de una parte de Egipto. En este caso Diodoro se está refiriendo al interior del pais y su transitabilidad natural, dado su carácter llano y poco accidentado, a las caballerias, lo cual no contradice su privilegiada geografia de pais fortificado naturalmente. Es ilustrativo un pasaje de las le've's donde la fortificación de las fronteras se combina con el cuidado de los caminos con el objeto de brindar fácil acceso y circulación a hombres y animales en el territorio (761 a). La recurrencia de la nota estratégica dentro de la presentación diodorea de Egipto es, una vez más, significativa. 